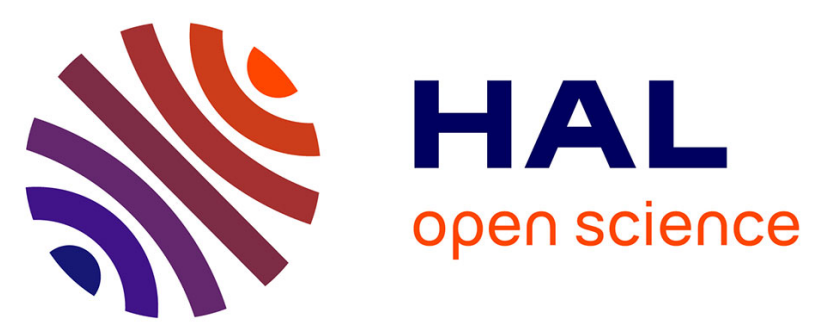

\title{
Influence of surface radiation on the transition to unsteadiness for a natural convection flow in a differentially heated cavity
}

\author{
P. Chorin, F. Moreau, Y. Billaud, D. Lemonnier, D. Saury
}

\section{- To cite this version:}

P. Chorin, F. Moreau, Y. Billaud, D. Lemonnier, D. Saury. Influence of surface radiation on the transition to unsteadiness for a natural convection flow in a differentially heated cavity. Numerical Heat Transfer, Part A Applications, 2020, 78 (7), pp.291 - 305. 10.1080/10407782.2020.1791550 . hal-03228339

\section{HAL Id: hal-03228339 \\ https://hal.science/hal-03228339}

Submitted on 18 May 2021

HAL is a multi-disciplinary open access archive for the deposit and dissemination of scientific research documents, whether they are published or not. The documents may come from teaching and research institutions in France or abroad, or from public or private research centers.
L'archive ouverte pluridisciplinaire HAL, est destinée au dépôt et à la diffusion de documents scientifiques de niveau recherche, publiés ou non, émanant des établissements d'enseignement et de recherche français ou étrangers, des laboratoires publics ou privés. 
archives-ouvertes

\title{
Influence of surface radiation on the transition to unsteadiness for a natural convection flow in a differentially heated cavity
}

\author{
P Chorin, F Moreau, Y Billaud, D Lemonnier, D Saury
}

\section{- To cite this version:}

P Chorin, F Moreau, Y Billaud, D Lemonnier, D Saury. Influence of surface radiation on the transition to unsteadiness for a natural convection flow in a differentially heated cavity. Numerical Heat Transfer, Part A Applications, Taylor \& Francis, 2020, 78, pp.291 - 305. 10.1080/10407782.2020.1791550 hal03228339

\section{HAL Id: hal-03228339 \\ https://hal.archives-ouvertes.fr/hal-03228339}

Submitted on 18 May 2021

HAL is a multi-disciplinary open access archive for the deposit and dissemination of scientific research documents, whether they are published or not. The documents may come from teaching and research institutions in France or abroad, or from public or private research centers.
L'archive ouverte pluridisciplinaire HAL, est destinée au dépôt et à la diffusion de documents scientifiques de niveau recherche, publiés ou non, émanant des établissements d'enseignement et de recherche français ou étrangers, des laboratoires publics ou privés. 


\title{
Influence of surface radiation on the transition to unsteadiness for a natural convection flow in a differentially heated cavity
}

\author{
P. Chorin (D), F. Moreau (D), Y. Billaud, D. Lemonnier (D), and D. Saury (D) \\ Institut Prime, UPR 3346 - CNRS $\diamond$ ENSMA $\diamond$ Université de Poitiers, Futuroscope Cedex, France
}

\begin{abstract}
The influence of surface radiation on the transition to the unsteady state in natural convection is studied numerically. The configuration of the differentially heated square cavity with adiabatic horizontal walls is chosen to generate an internal natural convection flow. It is known that radiative transfers reduce the temperature difference between the adiabatic walls, which consequently reduces the thermal stratification of the central zone and increases the velocity flow. Many studies have focused on the stationary regime, but few of them have investigated the transition to unsteady flow. For this purpose, the effect of the wall emissivity on the critical Rayleigh number and the associated critical frequency was studied for a given cavity length. The cavity length and mean temperature of isothermal walls are set for the whole study. The results show that all these values are between the values obtained without radiation and those obtained for perfectly conducting horizontal walls. The critical Rayleigh number decreases with emissivity while the associated frequency increases. Moreover, the symmetry of fluctuating properties of the flow is changed when the radiation is taken into account.
\end{abstract}

ARTICLE HISTORY

Received 3 May 2020

Accepted 30 June 2020

\section{Introduction}

The large variety of actual situations in which natural convection takes place (building ventilation, electronic devices, nuclear power plant, underhood space of cars, etc.) has led the scientific community to carry out numerous studies on the subject. In most of engineering fields, natural convection flow develops within confined spaces and the associated flow characteristics are complex. In order to better control the boundary conditions of the problem and thus facilitate its investigation, academic setups are commonly used. Among these setups, the differentially heated cavity (DHC), in which two opposite vertical walls are maintained at different temperatures, allows a thorough study of the flow and the associated heat transfers.

This configuration has been first studied by Batchelor [1]. As the analytical analysis of the problem is complex, numerical solutions have been proposed and several reference values are available $[2,3]$. Among the possible flow regimes in a DHC, those reference solutions concern the stationary flow. Later on, several authors have studied regime transitions that occur when the Rayleigh number increases [4-8]. This transition from steadiness to unsteadiness follows a supercritical Hopf bifurcation. If the Rayleigh number continues to increase, several other Hopf bifurcations occur which leads progressively the flow to chaos [8]. 


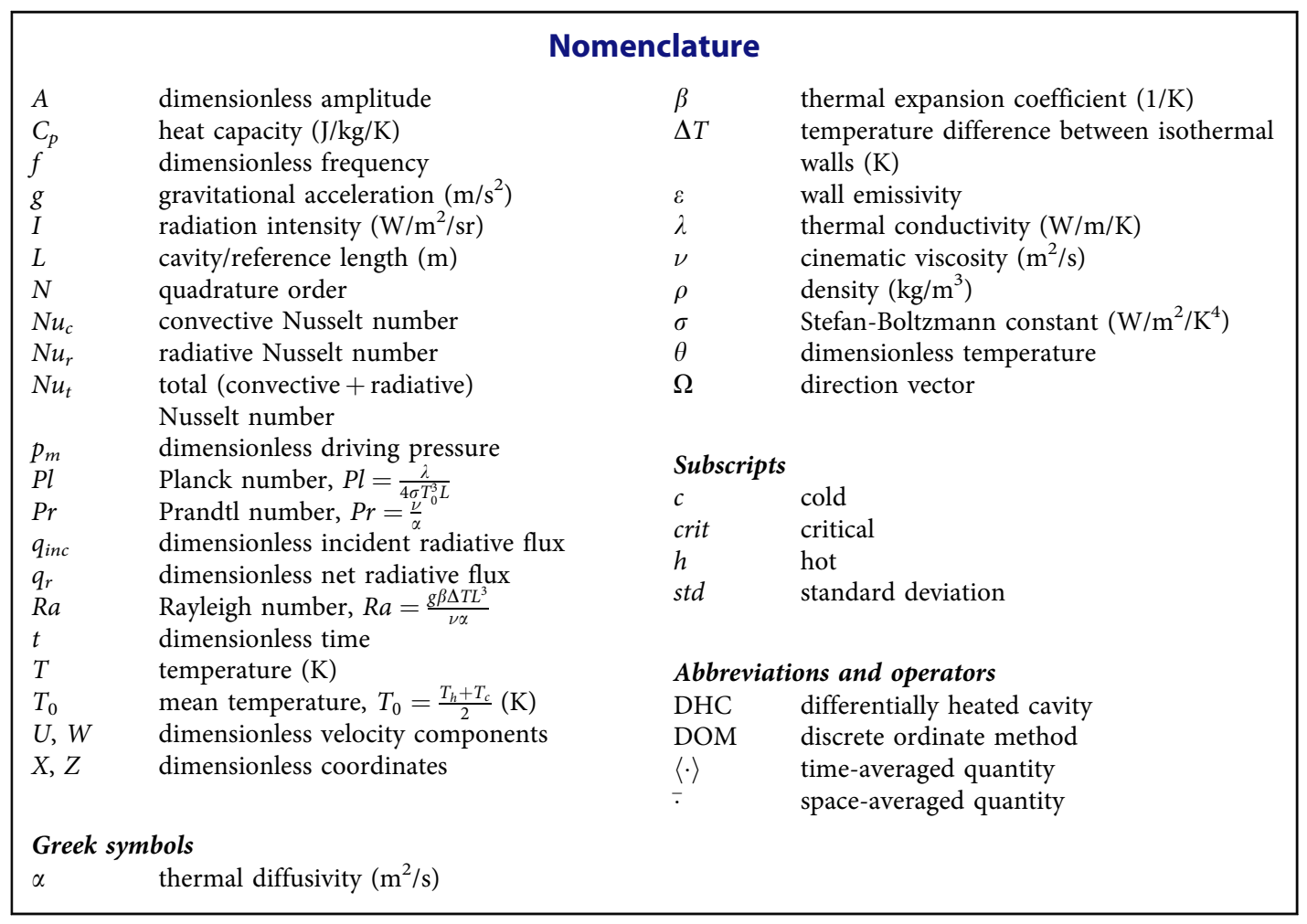

While some authors have focused on pure convective cases, others have begun to take into account radiative exchanges [9-12]. In these numerical studies, various radiative models (radiosity method, P1 approximation, discrete ordinate method...) are coupled to the convective model. Considering only surface to surface radiation (i.e. neglecting any radiative interaction within the medium), the radiative exchanges modify the temperature of the adiabatic walls, which acts both on the overall flow and on heat transfer. In particular, the thermal stratification of the central area is reduced. Consequently, a coupling between radiation and convection occurs through nonisothermal walls.

In those works, the analysis is conducted only on stationary flows. To our knowledge, the only study of the transition to the unsteady regime in the presence of surface radiation for a twodimensional cavity was carried out by Wang et al. [13]. Considering a square cavity and walls with emissivity of 0.2 , the authors have observed that emitting walls lead to trig earlier the transition to unsteadiness. In addition, the first frequency occurring in the flow is several times greater than in the absence of radiation.

The present work focuses on the transition to the unsteady state in the presence of surface radiation. A square cavity is considered in order to study the influence of the emissivity of the walls on the triggering of this transition and on the emerging frequency in the flow.

\section{Mathematical model}

The configuration mimics a differentially heated square cavity with isothermal vertical walls at temperatures $T_{h}$ and $T_{c}\left(\Delta T=T_{h}-T_{c}>0\right)$ and adiabatic horizontal walls (see Figure 1). The cavity length (used as reference length) is set to $L=0.335 \mathrm{~m}$. It is filled with atmospheric air whose thermophysical properties are taken constant except for the density (see below) and 


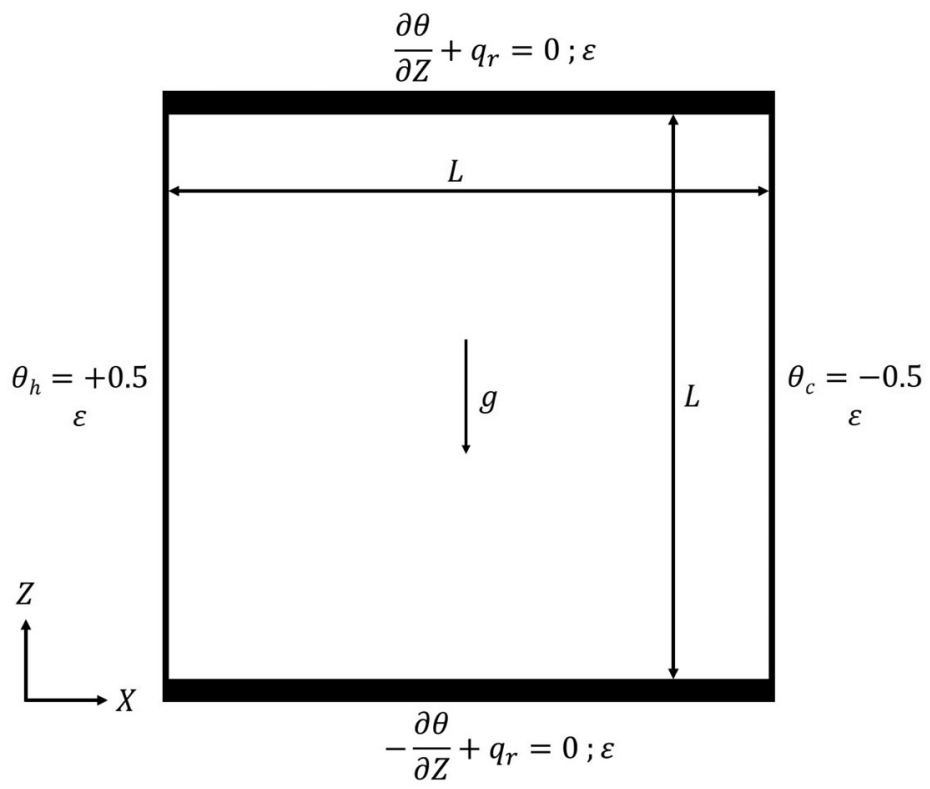

Figure 1. Scheme of the differentially heated square cavity.

evaluated at the mean cavity temperature $T_{0}=\frac{1}{2}\left(T_{h}+T_{c}\right)=293.15 \mathrm{~K}$. Under these conditions, the following fluid thermophysical properties are: Prandtl number $\operatorname{Pr}=0.711$, thermal conductivity $\lambda=0.0257 \mathrm{~W} \cdot \mathrm{m}^{-1} \cdot \mathrm{K}^{-1}$, thermal diffusivity $\alpha=2.115 \times 10^{-5} \mathrm{~m}^{2} \cdot \mathrm{s}^{-1}$ and heat capacity $C_{p}=$ $1005 \mathrm{~J} . \mathrm{kg}^{-1} . \mathrm{K}^{-1}$. The Boussinesq approximation is assumed, i.e. it is considered that the density is constant except in the buoyancy term where it linearly depends on temperature as following:

$$
\rho(T)=\rho_{0}\left[1-\beta\left(T-T_{0}\right)\right]
$$

where $\rho_{0}=1.207 \mathrm{~kg} \cdot \mathrm{m}^{-3}$ is the mean density and $\beta=3.41 \times 10^{-3} \mathrm{~K}^{-1}$ is the thermal expansion coefficient. The Rayleigh number indicates the flow regime and is defined as:

$$
R a=\frac{g \beta \Delta T L^{3}}{\nu \alpha}
$$

where $\nu$ is the cinematic viscosity. All variables relative to lengths, speeds, times and pressures are scaled using the following reference values: $L, \frac{\alpha}{L} \sqrt{R a}, \frac{L^{2}}{\alpha \sqrt{R a}}$ and $\rho_{0} \frac{\alpha^{2}}{L^{2}} R a$, respectively. Moreover, dimensionless temperature is written as $\theta=\frac{T-T_{0}}{\Delta T} \in[-0.5 ; 0.5]$. The dimensionless governing equations can then be written as follow:

$$
\begin{gathered}
\nabla \cdot \mathbf{V}=0 \\
\frac{\partial \mathbf{V}}{\partial t}+(\mathbf{V} . \boldsymbol{\nabla}) \mathbf{V}=-\nabla p_{m}+\operatorname{Pr} \theta \mathbf{e}_{\mathbf{z}}+\frac{\operatorname{Pr}}{\sqrt{R a}} \nabla^{2} \mathbf{V} \\
\frac{\partial \theta}{\partial t}+(\mathbf{V} . \nabla) \theta=\frac{1}{\sqrt{R a}} \nabla^{2} \theta
\end{gathered}
$$

where $\mathbf{e}_{\mathbf{z}}$ is the vector of vertical axis, oriented upwards. The associated boundary conditions in dimensionless form are:

- $U=W=0$ on all walls

- $\theta=\theta_{h}=+0.5$ and $\theta=\theta_{c}=-0.5$ on hot and cold walls, respectively

- $-\frac{\partial \theta}{\partial Z}+q_{r}=0$ and $\frac{\partial \theta}{\partial Z}+q_{r}=0$ on bottom and top walls, respectively 
Table 1. Convergence test on mesh size and relative gaps with reference values; $R a=10^{6}, \varepsilon=0.8, \Delta T=10 \mathrm{~K}$, on the hot wall.

\begin{tabular}{lcccc}
\hline Mesh & $90 \times 90$ & $110 \times 110$ & $130 \times 130$ & Wang et al. [13] \\
\hline$N u_{c}$ & 7.782 & 7.786 & 7.789 & 7.815 \\
$N u_{r}$ & $(-0.4 \%)$ & $(-0.4 \%)$ & $(-0.3 \%)$ & - \\
& 11.115 & 11.109 & 11.104 & 11.265 \\
\hline
\end{tabular}

where $q_{r}$ is the dimensionless net radiative flux defined as:

$$
q_{r}=\frac{\varepsilon}{4 P l} \frac{T_{0}}{\Delta T}\left[\left(\frac{T}{T_{0}}\right)^{4}-q_{i n c}\right]
$$

Due to the fluid properties mentioned previously, the Planck number $P l=\frac{\lambda}{4 \sigma T_{0}^{3} L}$ is consequently equal to $P l=1.34 \times 10^{-2}$ where $\sigma$ is the Stefan-Boltzmann constant. The dimensionless incident radiative flux $q_{i n c}$ is derived from:

$$
q_{i n c}=\frac{1}{\sigma T_{0}^{4}} \int_{n . \Omega<0} I(\mathbf{x}, \Omega)|n . \Omega| \mathrm{d} \Omega
$$

where $I$ is the radiation intensity and $\Omega$ the direction vector. All walls are radiatively active with the same emissivity $\varepsilon$. The convective and radiative Nusselt numbers on vertical walls are respectively defined as:

$$
\begin{aligned}
N u_{c} & =\int_{0}^{1} \frac{\partial \theta}{\partial X}(Z) \mathrm{d} Z \\
N u_{r} & =\int_{0}^{1} q_{r}(Z) \mathrm{d} Z
\end{aligned}
$$

Finally, the knowledge of convective and radiative Nusselt numbers let define the total Nusselt as:

$$
N u_{t}=N u_{c}+N u_{r}
$$

\section{Numerical methods and validation}

All computations are performed using the computational-fluid-dynamics software Code Saturne [14] based on a co-located finite-volume approach to discretize the Navier-Stokes and energy equations. A centered second-order scheme in space is used to solve the momentum and energy equations, whereas the time-advancement is achieved with a second-order Crank-Nicholson scheme. The time step is set to reach the CFL condition such that the Courant number is lower than 1 at any time and any location. The pressure-velocity coupling is carried out with a prediction-correction algorithm similar to SIMPLEC. A non-uniform mesh is used to obtain a wellrefined mesh near the walls, using a cell distribution in the two directions with the density function $d(m)=\cosh (4 m-2), m \in[0 ; 1]$.

The incident radiative flux is calculated using the discrete ordinate method (DOM) [15]. A set of discrete directions is defined, where each direction has its own weight according to a quadrature formulae. The $S_{N}$ quadrature is used here [16] where $\mathrm{N}$ is the quadrature order. This quadrature considers $N(N+2)$ directions. This computational routine was developed to this purpose and implemented in Code Saturne [17].

A mesh convergence study is carried out, based on comparison of Nusselt numbers. The values of convective and radiative Nusselt numbers at the hot wall for $R a=10^{6}$ (corresponding to a steady flow) and $\varepsilon=0.8$ are compared with results from Wang et al. [13]. The temperature 
Table 2. Convergence test on quadrature order and relative gaps with reference values; $R a=10^{6}, \varepsilon=0.8, \Delta T=10 \mathrm{~K}$, on the hot wall.

\begin{tabular}{lcccc}
\hline Order & $S_{4}$ & $S_{8}$ & $S_{12}$ & Wang et al. [13] \\
\hline$N u_{c}$ & 7.788 & 7.794 & 7.789 & 7.815 \\
& $(-0.3 \%)$ & $(-0.3 \%)$ & $(-0.3 \%)$ & - \\
$N u_{r}$ & 11.027 & 11.109 & 11.123 & 11.265 \\
& $(-2.1 \%)$ & $(-1.4 \%)$ & $(-1.3 \%)$ & - \\
\hline
\end{tabular}

Table 3. Comparison of total Nusselt numbers $N u_{t}$ of present work (PW) with values from Saravanan et al. [12] and Wang et al. [13], and relative gaps with reference values; $\Delta T=10 \mathrm{~K}$.

\begin{tabular}{lcccr}
\hline$N u_{t}$ & & & & \\
Ra & $\varepsilon$ & PW & $\begin{array}{c}\text { Saravanan } \\
\text { et al. [12] }\end{array}$ & Wang et al. [13] \\
\hline $10^{4}$ & 0.2 & 2.755 & $2.757(-0.1 \%)$ & $2.767(-0.4 \%)$ \\
& 0.8 & 4.602 & $4.629(-0.6 \%)$ & $4.650(-1.0 \%)$ \\
$10^{5}$ & 0.2 & 5.461 & $5.477(-0.3 \%)$ & $5.484(-0.4 \%)$ \\
& 0.8 & 9.298 & $9.371(-0.8 \%)$ & $9.385(-0.9 \%)$ \\
$10^{6}$ & 0.2 & 10.667 & $10.671(<0.1 \%)$ & $10.736(-0.6 \%)$ \\
& 0.8 & 18.902 & $19.029(-0.7 \%)$ & $19.080(-0.9 \%)$ \\
\hline
\end{tabular}
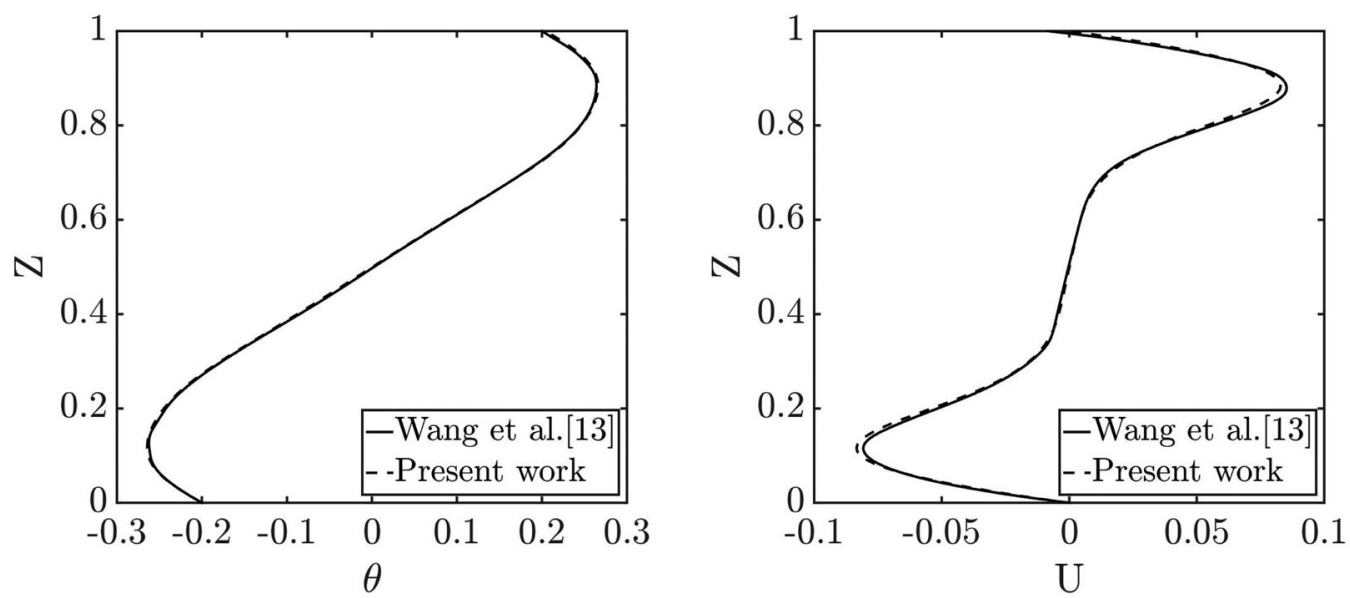

Figure 2. Temperature $\theta$ (left) and horizontal velocity component $U$ (right) vs. vertical location $Z$; comparison with results from Wang et al. [13]; $R a=10^{6}, \varepsilon=0.2$.

difference between the isothermal walls is set to $\Delta T=10 \mathrm{~K}$, which is associated in that validation case with a cavity length of $L=0.983 \mathrm{~m}$. It can be seen in Table 1 that for the three meshes considered, the relative gaps on the Nusselt numbers calculated with the reference case are smaller than $1.4 \%$ in absolute value. However, the mesh of size $90 \times 90$ does not allow to detect the unsteady behavior of the flow with a higher Rayleigh number, due to the occurrence of erratic oscillations. That is why the mesh $110 \times 110$ is retained thereafter.

Convergence on quadrature order for the same test case considering quadratures $S_{4}, S_{8}$ and $S_{12}$ (having 24, 80 and 168 directions, respectively) is displayed in Table $2 . N u_{c}$ values are very close whatever the quadrature order. However, the relative gap on $N u_{r}$ for quadrature $S_{4}$ is larger than those for quadratures $S_{8}$ and $S_{12}$ (which are close to each other). Thus the $S_{8}$ quadrature is retained as it offers the best compromise between accuracy and computational time.

The total Nusselt numbers $N u_{t}$ obtained for $R a=\left(10^{4}, 10^{5}, 10^{6}\right)$ and $\varepsilon=(0.2,0.8)$ are compared to results from Saravanan and Sivaraj [12] and Wang et al. [13] in Table 3. The flow is steady for all considered $R a$. As in [12,13], the temperature difference between isothermal walls is set at $\Delta T=10 \mathrm{~K}$ and the cavity length is adapted according to the Rayleigh number. Relative 


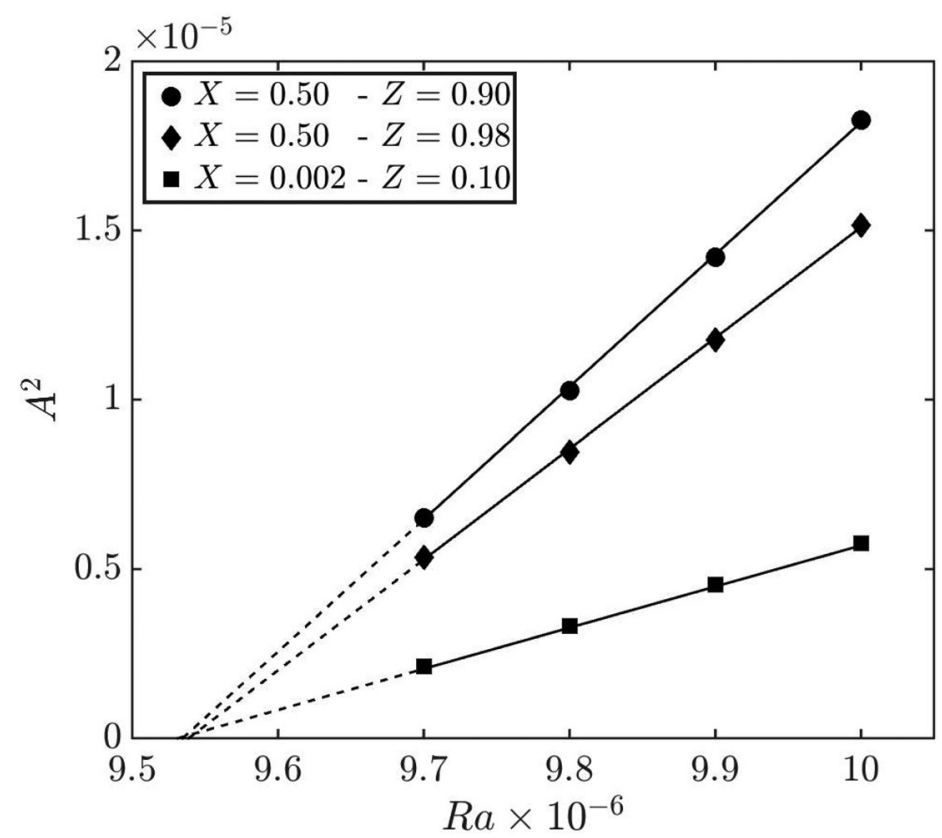

Figure 3. Determination of the critical Rayleigh number $R a_{\text {crit }}$ based on the linear variation of the squared amplitude $A^{2}$ with $R a$.
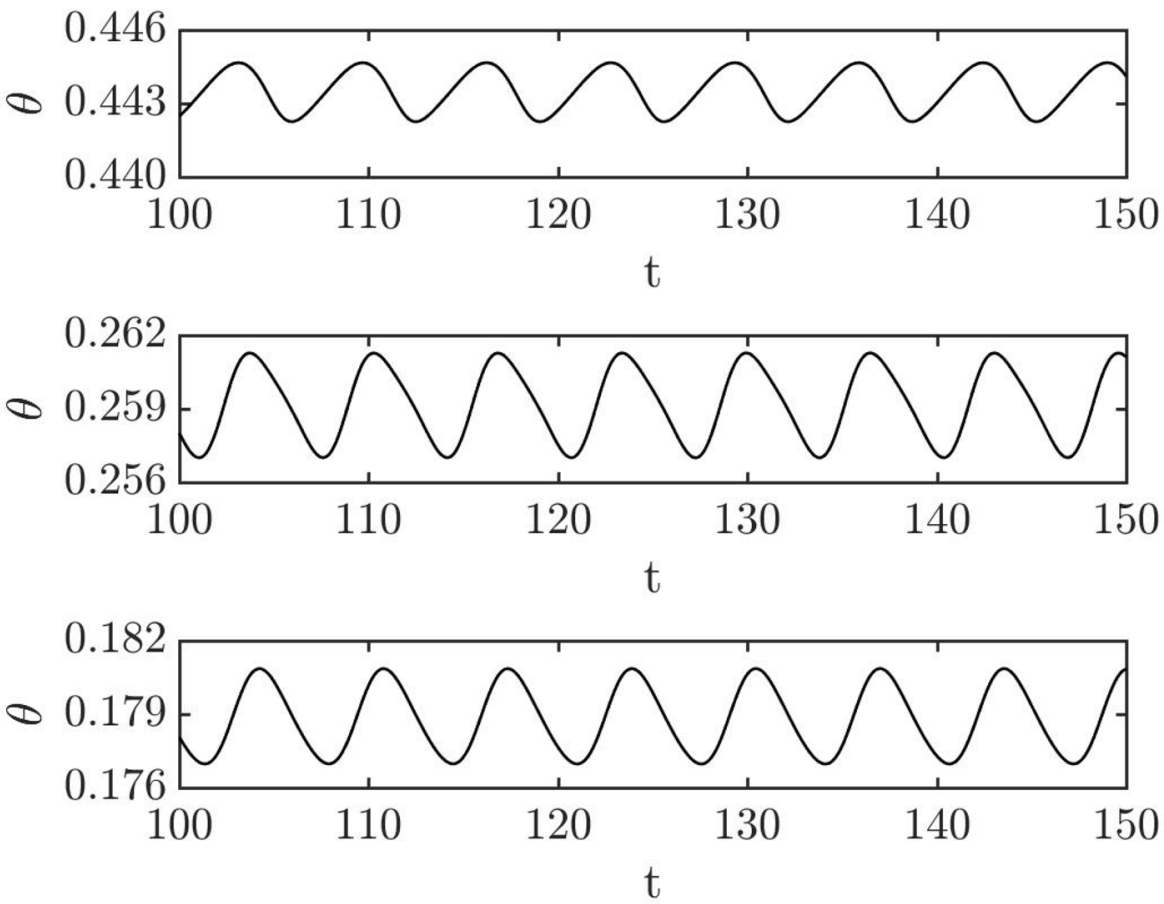

Figure 4. Temporal evolution of temperature $\theta$ at $R a=10^{7}$ from an arbitrary time $t_{0}=100$; (top) $X=0.002$ and $Z=0.10$ (middle) $X=0.50$ and $Z=0.90$ (bottom) $X=0.50$ and $Z=0.98$. 
Table 4. Comparison of critical Rayleigh numbers $R a_{\text {crit }}$ and critical frequencies $f_{\text {crit }}$ from present work with results from Wang et al. [13]; $\mathrm{Pl}=1.34 \times 10^{-2}$.

\begin{tabular}{lccc}
\hline & & Wang et al. [13] & Present Work \\
\hline$R a_{\text {crit }}$ & $\varepsilon=0$ & $1.82 \times 10^{8}$ & $1.81 \times 10^{8}(-0.6 \%)$ \\
$f_{\text {crit }}$ & $\varepsilon=0.2$ & $9.3 \times 10^{6}$ & $9.5 \times 10^{6}(+2.5 \%)$ \\
& $\varepsilon=0$ & 0.047 & $0.045(-2.6 \%)$ \\
& $\varepsilon=0.2$ & 0.154 & $0.153(-0.4 \%)$ \\
\hline
\end{tabular}

gaps are below or equal to $1.0 \%$. In addition, the profiles of temperature and horizontal component of velocity at mid-width $(X=0.50)$ for the case $R a=10^{6}$ and $\varepsilon=0.2$ are given in Figure 2 . There is a good agreement with the profiles from [13]. The code is thus validated thanks to the reference results for steady cases.

It must also be checked whether the transition from steady to unsteady state occurs at the correct critical Rayleigh number $R a_{\text {crit }}$ and with the correct flow frequency $f_{\text {crit }}$. To our knowledge, the only study of this transition for a bidimensional cavity submitted to radiation is that of Wang et al. [13]. The authors chose the particular value of $L=0.335 \mathrm{~m}$ and a wall emissivity of $\varepsilon=0.2$. As the transition is following a supercritical Hopf bifurcation, the squared amplitude of the fluctuations evolves linearly with $R a$ in the vicinity of $R a_{\text {crit }}$. This relationship is used to estimate the value of the critical Rayleigh number (see Figure 3). The measurement points have been chosen such that the temporal evolution of the temperature signals is almost sinusoidal (see Figure 4), which is a necessary condition for this relation to be verified. Indeed, in some points in the cavity the temporal signals are more strongly modified, although the fundamental frequency is the same. As seen in Table 4, the $R a_{\text {crit }}$ and $f_{\text {crit }}$ values with and without radiative transfer are in good agreement with the ones in the literature. This demonstrates the capability of the numerical methods used in the present work to properly detect the transition to unsteadiness.

\section{Results and discussions}

In order to study natural convection with the interaction of surface radiation in DHC, the cavity length has to be set because the effects of radiation depend on it (see [17]). The cavity length is set at $L=0.335 \mathrm{~m}$ [13] and $T_{0}$ is maintained at $293.15 \mathrm{~K}$, corresponding to a Planck number $P l=$ $1.34 \times 10^{-2}$. This ensure relatively small values of $\Delta T$ (from 1 to $15 \mathrm{~K}$ ), which allows to stay within the Boussinesq approximation. For cases without radiation, $R a$ is the unique driving parameter and the cavity length can be chosen such that $\Delta T \leq 15 \mathrm{~K}$.

In order to illustrate the effects of radiation, temperature fields for $\varepsilon=0$ (without radiation) and $\varepsilon=1$ (black surfaces) at $R a=10^{6}$ are given in Figure 5. The streamlines are superimposed in order to visualize the associated structure of the flow. Without considering wall radiation (i.e. $\varepsilon=0)$, the flow crosses the central area $(X \in[0.4,0.6])$ mainly through horizontal flowlines. When black surface radiation is considered (i.e. $\varepsilon=1$ ), the flow exhibits major vortex structures, in particular a large central vortex with a direction of rotation in opposition to the one of the main flow, which has not been previously noticed in the literature to the authors' knowledge. These changes on core flow are highlighted in Figure 6 with the profiles of the horizontal component of velocity $U$ against the elevation $Z$ at mid-with of the cavity. The maximum of the horizontal velocity is more than twice higher for $\varepsilon=1$ than for $\varepsilon=0(0.132$ against 0.065$)$ whereas the reverse flow (contra-rotating vortex) is observed between $Z=0.3$ and $Z=0.7$ only for $\varepsilon=1$. Consequently, the flow rate on the mid-height of the cavity at $X=0.5$ is $12 \%$ higher with wall radiation. Moreover, it can be noticed on the temperature fields (Figure 5) that the central area has an important thermal stratification in the case where wall radiation is not considered $(\varepsilon=0)$ but a weaker one in the case of wall radiation with black surfaces. The thermal stratification at the center of the cavity $S=\frac{\partial \theta}{\partial Z}(X=0.5 ; Z=0.5)$ is thus $26 \%$ lower for $\varepsilon=1(S=0.680)$ than for $\varepsilon=0 \quad(S=0.916)$. This difference in thermal stratification is linked to a change in the 

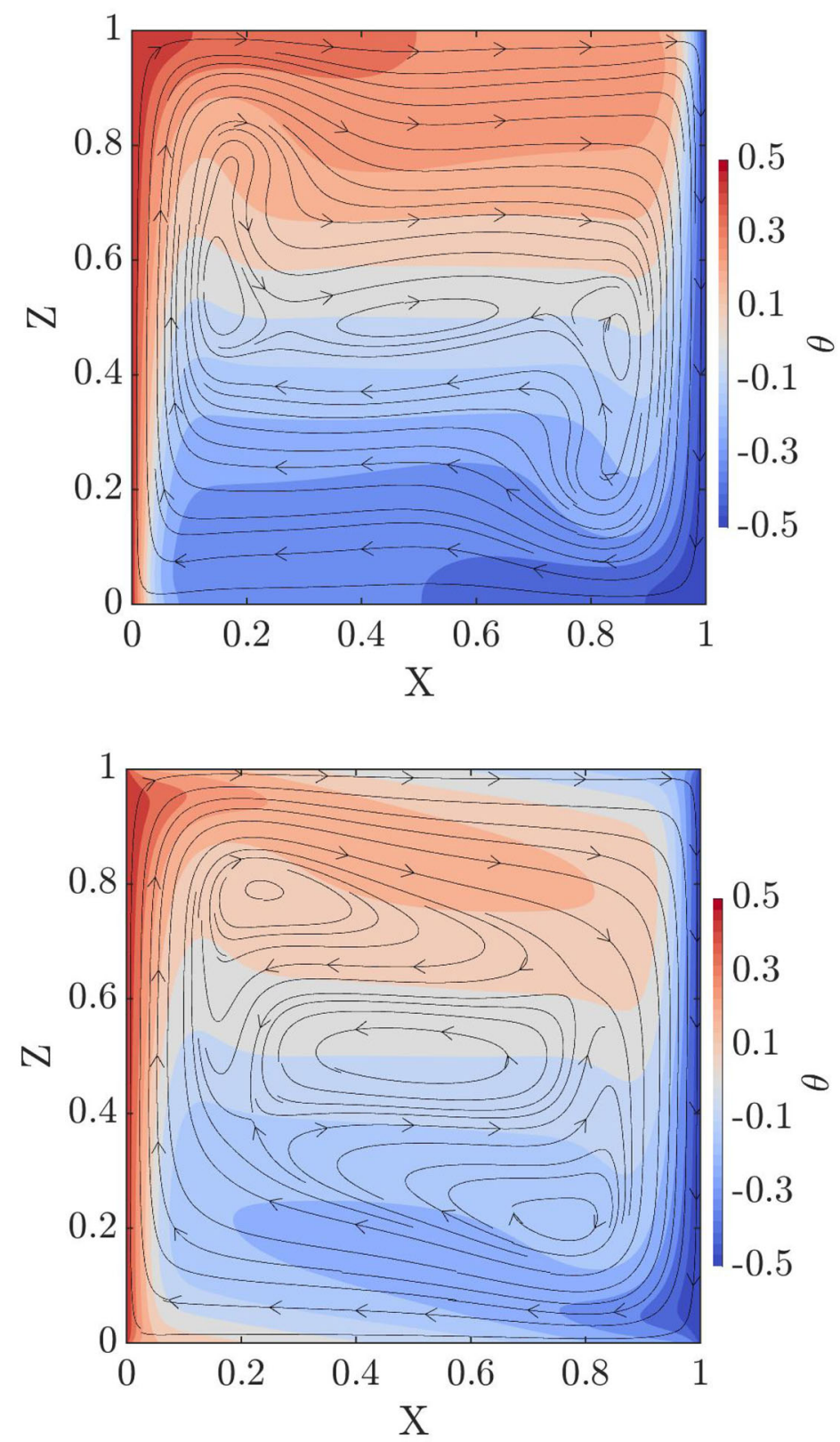

Figure 5. Temperature fields for $R a=10^{6}, P I=1.34 \times 10^{-2}$; (top) $\varepsilon=0$ and (bottom) $\varepsilon=1$; streamlines are overprinted.

temperature of the adiabatic walls: the radiation exchange reduces the temperature difference between the top and bottom adiabatic walls and therefore the stratification within the cavity (which in turn modifies the structure of the flow). This change in temperature distribution on the adiabatic walls for these two extreme cases is shown in Figure 7. For $\varepsilon=0$, the mean temperature difference between the top and bottom walls is equal to 0.601 , that means $60.1 \%$ of the temperature difference between the isothermal walls, against only $6.5 \%$ for $\varepsilon=1$.

Thus the radiation exchanges lead the present case, with adiabatic horizontal walls, close to the case of perfectly conducting horizontal walls for which the temperature difference between these walls is zero. As noted by Wang et al. [13], it can be expected that the critical Rayleigh 


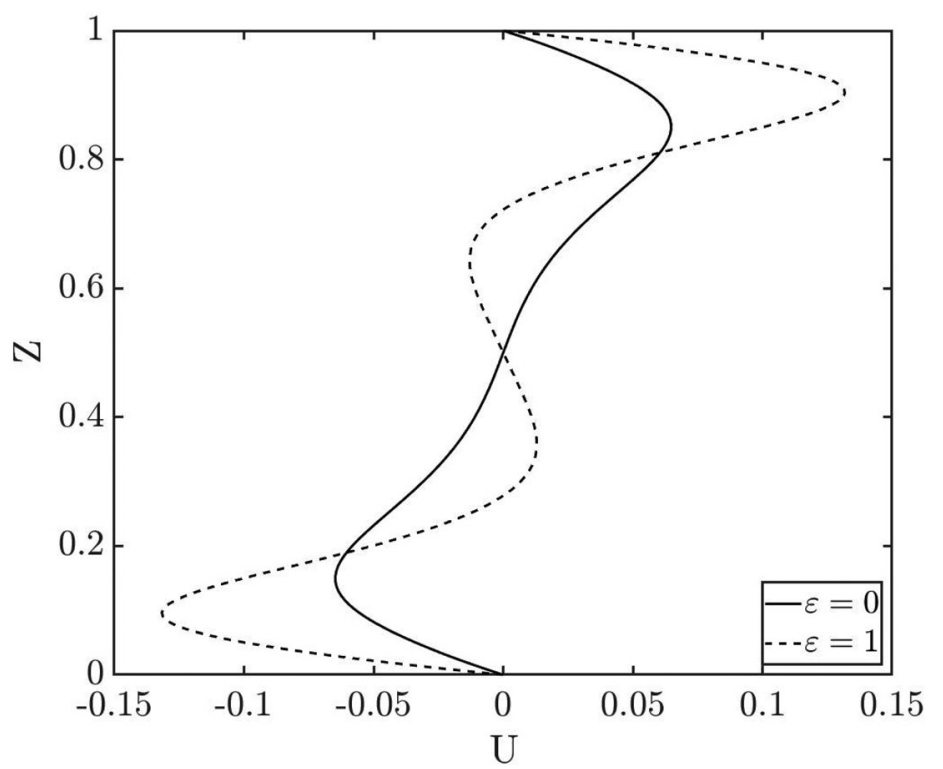

Figure 6. Horizontal velocity component $U$ profiles at $X=0.5, R a=10^{6}$ and $P I=1.34 \times 10^{-2}$ for $\varepsilon=0$ and $\varepsilon=1$.

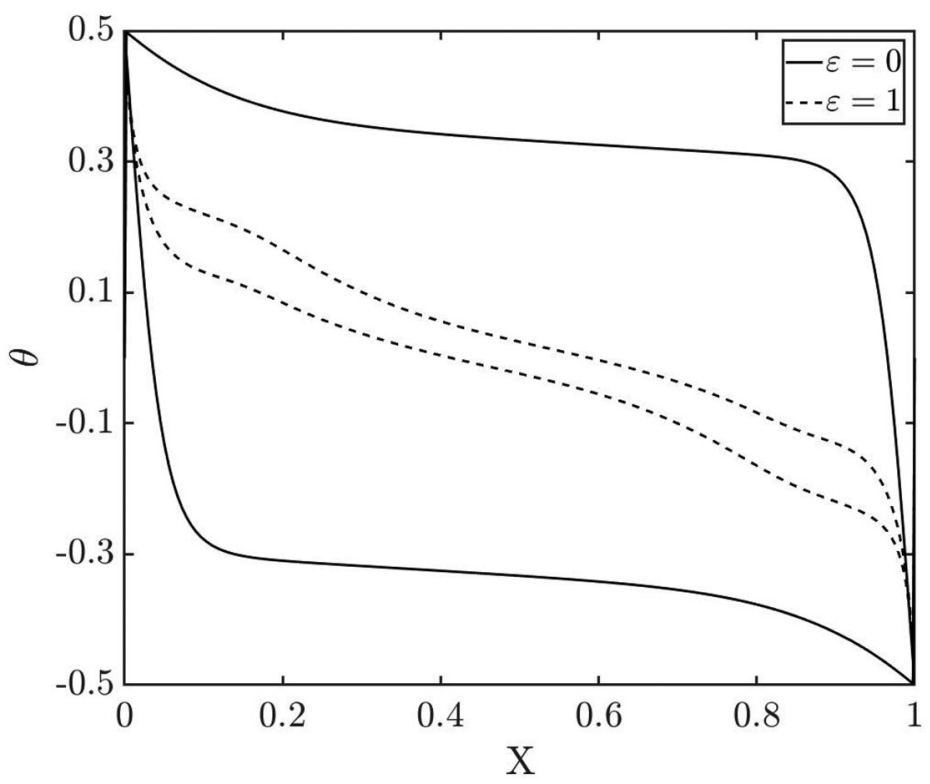

Figure 7. Temperature profiles at top and bottom walls at $R a=10^{6}$ and $P I=1.34 \times 10^{-2}$ for $\varepsilon=0$ and $\varepsilon=1$.

number of the onset of the unsteady state is then intermediate between the value for adiabatic walls in pure convection $\left(R a_{c r i t}=1.82 \times 10^{8}\right)$ and the value for perfectly conducting horizontal walls $\left(R a_{\text {crit }}=2.11 \times 10^{6}\right)$. In addition, it can also be expected that the emerging frequency in the flow has a value between the adiabatic case without radiation $\left(f_{\text {crit }}=0.046\right)$ and the one for perfectly conducting horizontal walls $\left(f_{\text {crit }}=0.214\right)$. In order to check these assumptions and to determine how these quantities vary in presence of radiation, the critical Rayleigh number and the critical frequency as functions of the emissivity are given in Figure 8 . The frequencies are obtained using spectral analysis (see insert in Figure 8). All the values of $R a_{\text {crit }}$ and $f_{\text {crit }}$ are actually between the limits previously expected. Moreover, these quantities vary monotonically and 


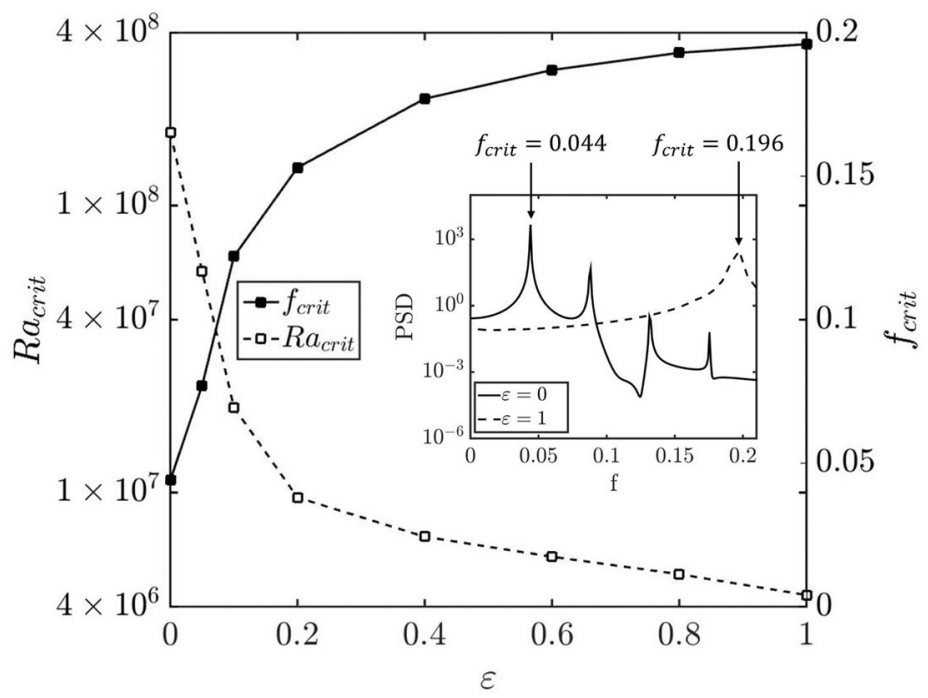

Figure 8. Critical Rayleigh number $R a_{\text {crit }}$ and critical frequency $f_{\text {crit }} v s$. wall emissivity $\varepsilon$ for $P I=1.34 \times 10^{-2} ; R a_{\text {crit }}$ is displayed in logarithmic scale and $f_{\text {crit }}$ is displayed in linear scale; Power Spectral Densities for $\varepsilon=0$ and $\varepsilon=1$ are inserted.

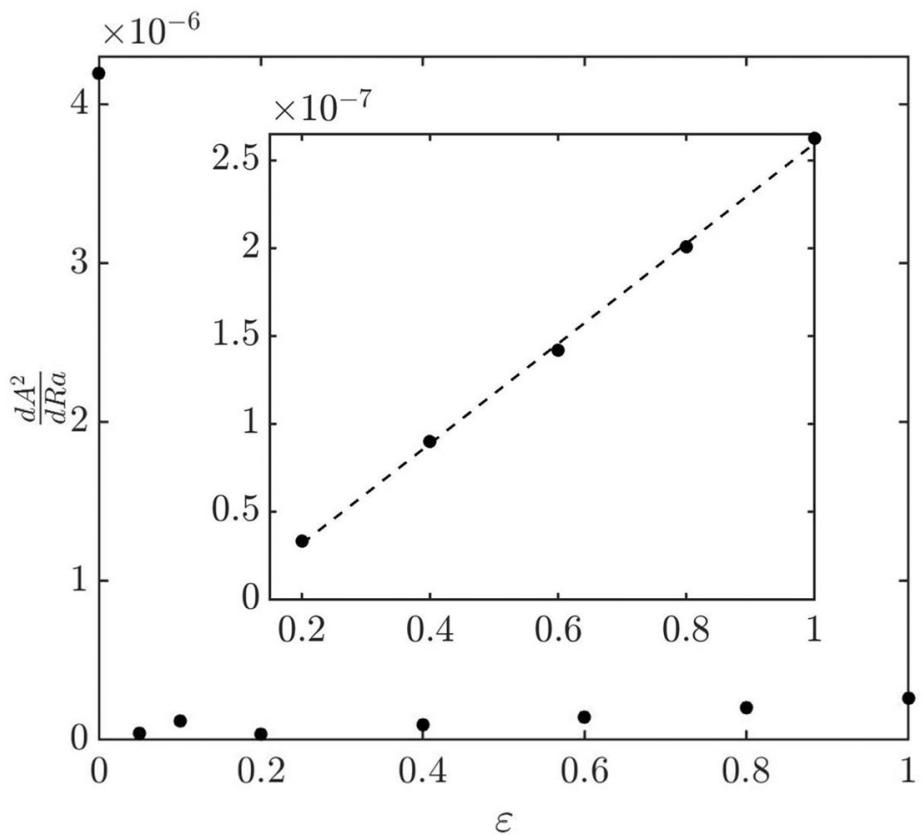

Figure 9. Growth rate of the squared amplitude of temperature fluctuation with the Rayleigh number $\frac{d A^{2}}{d R a}$ at $(X=0.508 ; Z=0.508)$ vs. wall emissivity $\varepsilon$ for $P I=1.34 \times 10^{-2}$; specific cases for $\varepsilon \geq 0.2$ are inserted with a linear regression for $\varepsilon \in[0.2 ; 1]$.

for low emissivities the variations of $R a_{\text {crit }}$ and $f_{\text {crit }}$ are greater than for high emissivities, as the slopes decrease progressively when $\varepsilon$ increases. $f_{\text {crit }}$ is thus almost four times higher for $\varepsilon=1$ than for $\varepsilon=0$ while $R a_{\text {crit }}$ has decreased by two orders of magnitude.

Another characteristic of the onset of the unsteady state is the growth rate of the fluctuations from the critical Rayleigh number. As it was seen in Figure 3, the square of the amplitude of the temperature fluctuations $A^{2}$ at any point inside the cavity growths linearly with $R a$. The growth 

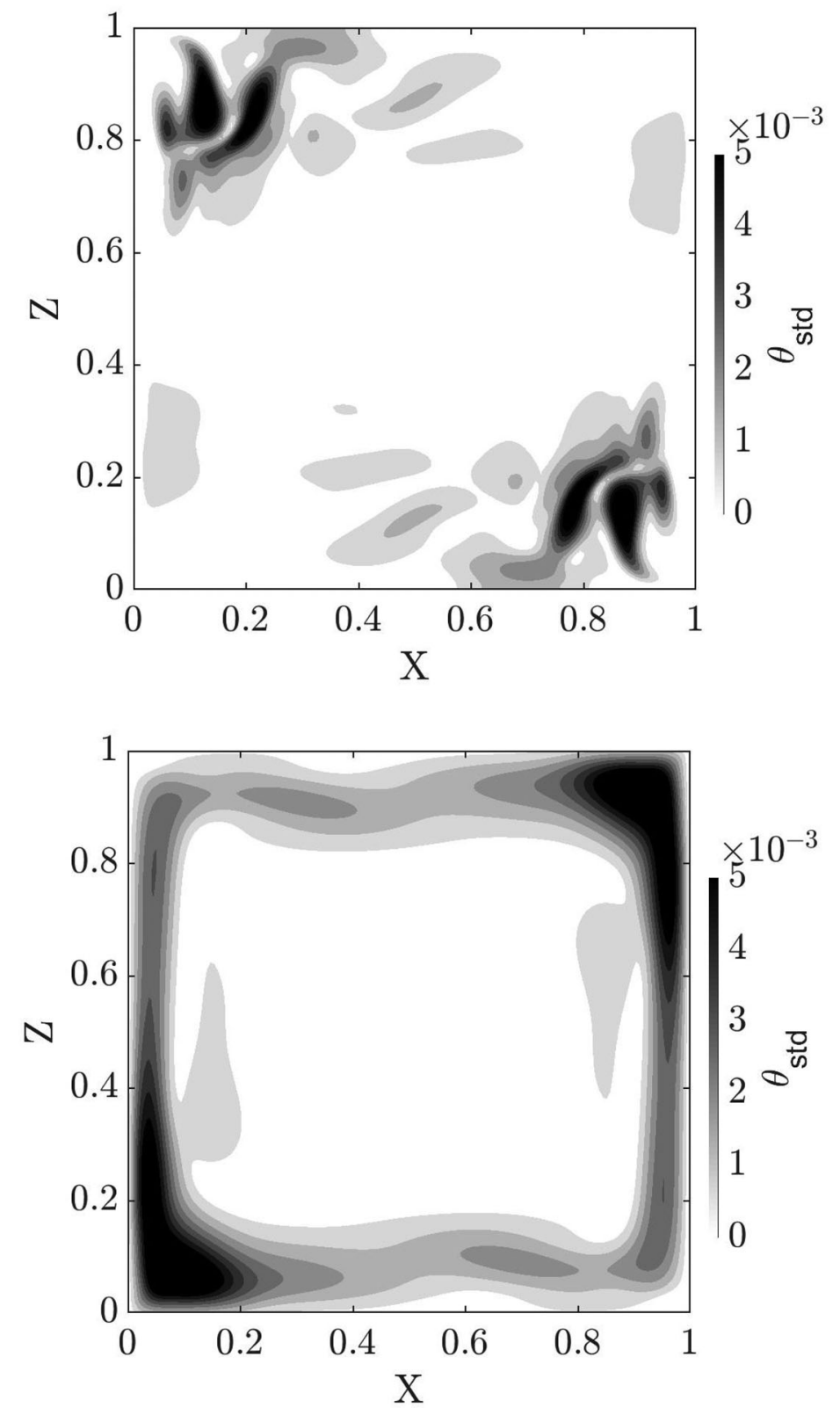

Figure 10. Standard deviation on temperature $\theta_{\text {std }}$ for $P I=1.34 \times 10^{-2}$; (top) $\varepsilon=0$ and $R a=1.82 \times 10^{8}$, (bottom) $\varepsilon=1$ and $R a=4.5 \times 10^{6}$.

rate of these fluctuations $\frac{d A^{2}}{d R a}$ is thus the slope of the profile of $A^{2}$ against $R a$. The variation of $\frac{d A^{2}}{d R a}$ measured on the temperature at $(X=0.508 ; Z=0.508)$ against $\varepsilon$ is shown in Figure 9. The value for the case without wall radiation $(\varepsilon=0)$ is one order of magnitude higher than the values for the cases with wall radiation $(\varepsilon \neq 0)$, hence the profile for $\varepsilon \geq 0.2$ is inserted. $\frac{d A^{2}}{d R a}(\varepsilon)$ starts by strongly decreasing for $\varepsilon \approx 0$ and then increases in a linearly for emissivities greater than 0.2 . Indeed, the square of the correlation coefficient obtained for the linear regression in the range $\varepsilon \in[0.2 ; 1]$ is greater than 0.999 . Even if this first decrease following by an increase on this profile is observed at the other measuring points, a linear relationship is not clearly observed in other regions of the cavity. This behavior can be linked to the changes of the Rayleigh number and 

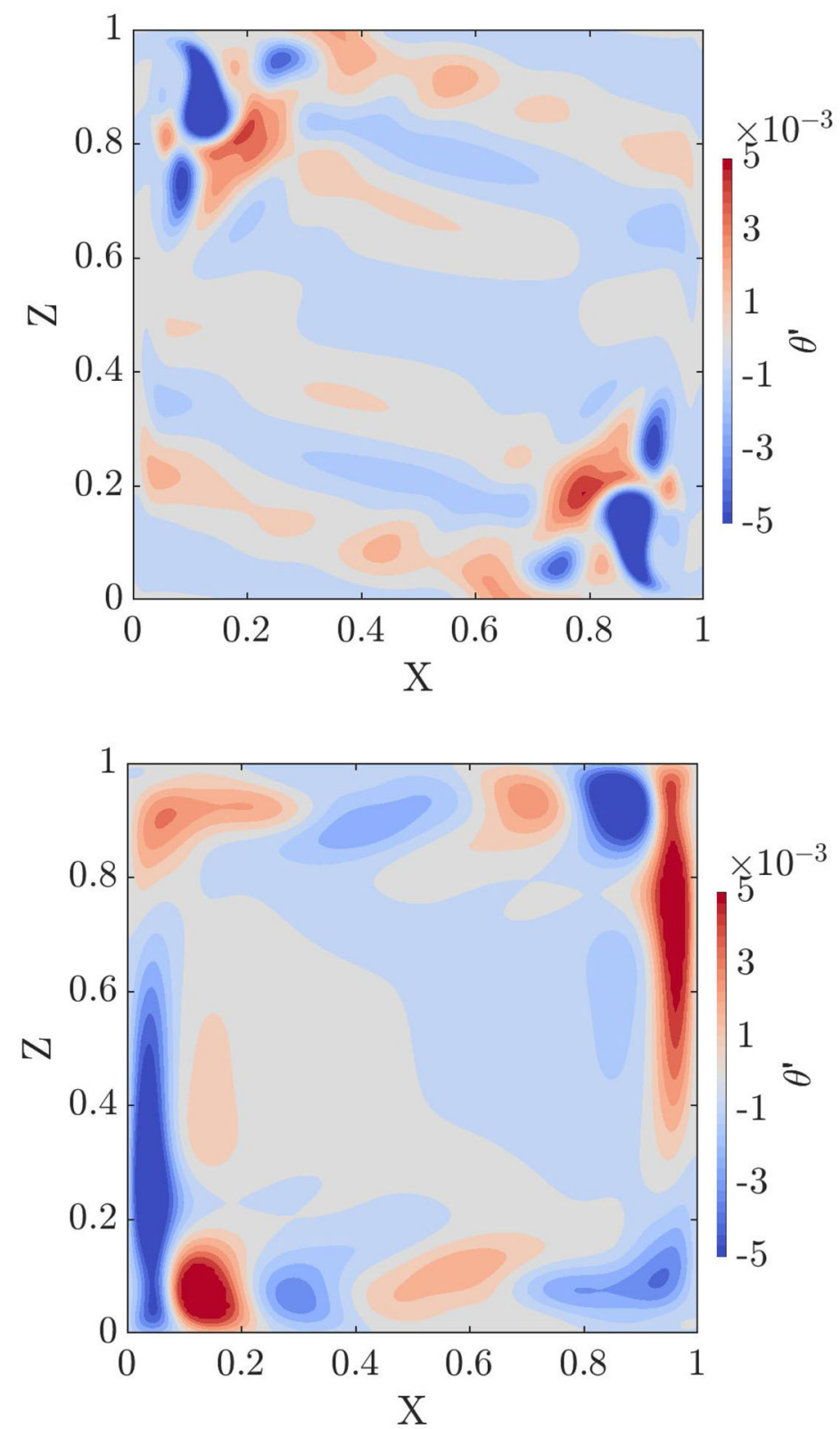

Figure 11. Fluctuation of temperature $\theta \prime$ for $P I=1.34 \times 10^{-2}$; (top) $\varepsilon=0$ and $R a=1.82 \times 10^{8}$, (bottom) $\varepsilon=1$ and $R a=$ $4.5 \times 10^{6}$ at an arbitrary time.

thermal stratification for the tested wall emissivities, which modifies the mean flow, the temperature distribution and consequently the growth rate of fluctuations. As this point is closed to the center of the cavity i.e. the symmetry point of the mean flow, it is less impacted by these changes.

In order to observe the distribution of the fluctuations within the cavity, standard deviations on temperature at the beginning of the supercritical regime for both the cases $\varepsilon=0$ and $\varepsilon=1$ are plotted in Figure 10. It is worth mentioning that, in this figure, the displayed Rayleigh number depends on the considered case since the critical Rayleigh number changed with wall emissivities. In the absence of wall radiation $(\varepsilon=0)$, the temperature fluctuations are concentrated at the 
Table 5. Numerical results slightly below the $R a_{\text {crit }}$ value for each tested emissivity $\varepsilon$ : thermal stratification $S$, mean temperatures of the bottom and top walls $\theta_{(Z=0)}$ and $\theta_{(Z=1)}$, convective and radiative Nusselt numbers $N u_{c}$ and $N u_{r}$ on the hot wall $(X=0)$ and on the cold wall $(X=1)$.

\begin{tabular}{lccccccrr}
\hline$\varepsilon$ & $R a$ & $S$ & $\theta_{(Z=0)}^{-}$ & $\theta_{(Z=1)}^{-}$ & $N u_{c, X=0}$ & $N u_{c, X=1}$ & $N u_{r, X=0}$ & $N u_{r, X=1}$ \\
\hline 0 & $1.8 \times 10^{8}$ & 1.012 & -0.366 & 0.366 & 35.211 & 35.228 & & \\
0.05 & $6.0 \times 10^{7}$ & 0.983 & -0.293 & 0.296 & 25.849 & 25.898 & 1.908 & 1.866 \\
0.1 & $1.9 \times 10^{7}$ & 0.938 & -0.232 & 0.234 & 18.613 & 18.641 & 3.831 & 3.803 \\
0.2 & $9.6 \times 10^{6}$ & 0.882 & -0.166 & 0.168 & 15.053 & 15.080 & 7.842 & 7.814 \\
0.4 & $6.6 \times 10^{6}$ & 0.805 & -0.103 & 0.105 & 13.188 & 13.218 & 16.560 & 16.528 \\
0.6 & $5.6 \times 10^{6}$ & 0.763 & -0.073 & 0.075 & 12.433 & 12.465 & 26.407 & 26.372 \\
0.8 & $5.0 \times 10^{6}$ & 0.740 & -0.056 & 0.058 & 11.905 & 11.929 & 37.005 & 36.969 \\
1 & $4.2 \times 10^{6}$ & 0.721 & -0.043 & 0.045 & 11.237 & 11.263 & 49.507 & 49.474 \\
\hline
\end{tabular}

end of the vertical boundary layers as observed by Le Quéré and Behnia [6]. On the other hand, when wall radiative exchanges occur, the largest amplitudes of the fluctuations are located at the beginning of the vertical boundary layers (and with lower amplitudes along all the walls, region where the mean flow velocity is large).

Symmetry is another physical property used to characterize unsteady flows. In a DHC with pure convection (i.e. $\varepsilon=0$ ), the mean flow is always centro-symmetric (CS), which means that it satisfies for each $(X, Z)$ locations the following equation:

$$
\langle\phi\rangle(1-X, 1-Z)=\delta\langle\phi\rangle(X, Z)
$$

with $\phi=(U, W, \theta)$ and $\delta=-1$.

In addition, the fluctuating flow obtained by replacing $\langle\phi\rangle$ by $\phi^{\prime}=\phi-\langle\phi\rangle$ can be centrosymmetric if $\delta=-1$ or anti-centro-symmetric (ACS) if $\delta=1$, according to the unsteady mode considered [8]. In the absence of wall radiation $(\varepsilon=0)$, the first unsteady mode is ACS [8]. The results in this study show that the first mode obtained when surface radiation is considered is CS whatever the value of the emissivity. This CS behavior was also found for a cavity with perfectly conducting horizontal walls. Thus the presence of wall radiation modifies the symmetry of the fluctuating temperature field. This difference can be seen in Figure 11: the fluctuating structures are in phase in Figure 11 (top) but in phase opposition in Figure 11 (bottom) with respect to the center of the cavity.

Finally, for information, several data obtained during this study are gathered and made available in Table 5.

\section{Conclusion}

A natural convection flow in a differentially heated square cavity with adiabatic walls is considered. The working fluid is air taken at normal condition of temperature. The solutions are obtained numerically by finite volume method. A radiative model is solved by the discrete ordinate method allowing to take into account radiative exchanges between the walls. The influence of the wall emissivity on the triggering of the transition from stationary to unsteady state has been studied for a cavity of length $0.335 \mathrm{~m}$ and for a mean temperature cavity $T_{0}=293.15 \mathrm{~K}$. The following conclusions can be drawn:

- All critical Rayleigh numbers and emerging frequencies in the flow are between the values of the case without radiation and the values of the case with perfectly conducting horizontal walls.

- Critical Rayleigh number decreases with the emissivity while the frequency appearing in the flow increases. 
- The growth rate of the square of the amplitude of the temperature fluctuations close to the center of the cavity strongly decreases first for very low wall emissivities and then increases in a linear way for emissivities larger emissiviies $(\varepsilon>0.2)$.

- In the case where wall radiation is considered $(\varepsilon \neq 0)$, temperature fluctuations are mainly located inside the boundary layers.

- Wall radiation changes the symmetry of the fluctuating temperature field, especially the first unsteady mode becomes centro-symmetric, whereas it was anti-centro-symmetric when wall emissivity is null.

\section{Acknowledgments}

The authors would also like to thank X. B. Nguyen for fruitful discussions.

\section{Funding}

The authors acknowledge the CPER (2015-2020) and ERDF (2014-2020) programs for supporting this work through the funding of equipments.

\section{ORCID}

P. Chorin (iD) http://orcid.org/0000-0003-3867-0504

F. Moreau iD http://orcid.org/0000-0002-4746-3534

D. Lemonnier (iD http://orcid.org/0000-0003-0508-3979

D. Saury (D) http://orcid.org/0000-0002-8035-5041

\section{References}

[1] G. K. Batchelor, "Heat transfer by free convection accross a closed cavity between vertical boundaries at different temperatures," Quart. Appl. Math., vol. 12, no. 3, pp. 209-233, 1954. DOI: 10.1090/qam/64563.

[2] G. De Vahl Davis and I. P. Jones, "Natural convection in a square cavity: a comparison exercise," Int. J. Numer. Meth. Fluids, vol. 3, no. 3, pp. 227-248, 1983. DOI: 10.1002/fld.1650030304.

[3] P. Le Quéré, "Accurate solutions to the square thermally driven cavity at high Rayleigh number," Computers Fluids, vol. 20, no. 1, pp. 29-41, 1991. DOI: 10.1016/0045-7930(91)90025-D.

[4] S. Paolucci and D. Chenoweth, "Transition to chaos in a differentially heated vertical cavity," J. Fluid Mech., vol. 201, no. 1, pp. 379-410, 1989. APR DOI: 10.1017/S0022112089000984.

[5] S. Armfield and J. C. Patterson, "Direct simulation of wave interactions in unsteady natural convection in a cavity," Int. J. Heat Mass Transfer, vol. 34, no. 4/5, pp. 929-940, 1991. DOI: 10.1016/0017-9310(91)90004-X.

[6] P. Le Quéré and M. Behnia, "From onset of unsteadiness to chaos in a differentially heated square cavity," J. Fluid Mech., vol. 359, pp. 81-107, 1998. DOI: 10.1017/S0022112097008458.

[7] E. Gadoin, P. Le Quéré, and O. Daube, "A general methodology for investigating flow instabilities in complex geometries: application to natural convection in enclosures," Int. J. Numer. Meth. Fluids, vol. 37, no. 2, pp. 175-208, 2001. SEP 30 DOI: 10.1002/fld.173.

[8] S. Xin and P. Le Quéré, "Natural-convection flows in air-filled, differentially heated cavities with adiabatic horizontal walls," Numer. Heat Transfer A, vol. 50, no. 5, pp. 437-466, 2006. SEP 15 DOI: 10.1080/ 10407780600605039.

[9] M. Behnia, J. Reizes, and G. de Vahl Davis, "Combined radiation and natural convection in a rectangular cavity with a transparent wall and containing a non-participating fluid," Int. J. Numer. Meth. Fluids, vol. 10, no. 3, pp. 305-325, 1990. DOI: 10.1002/fld.1650100306.

[10] M. Akiyama and Q. Chong, "Numerical analysis of natural convection with surface radiation in a square enclosure," Numer Heat Transfer, A: Appl., vol. 32, no. 4, pp. 419-433, 1997. DOI: 10.1080/ 10407789708913899.

[11] H. Bouali, A. Mezrhab, H. Amaoui, and M. Bouzidi, "Radiation-natural convection heat transfer in an inclined rectangular enclosure," Int. J. Thermal Sci., vol. 45, no. 6, pp. 553-566, 2006. DOI: 10.1016/j.ijthermalsci.2005.10.001. 
[12] S. Saravanan and C. Sivaraj, "Coupled thermal radiation and natural convection heat transfer in a cavity with a heated plate inside,” int. j. Heat Fluid Flow, vol. 40, pp. 54-64, 2013. DOI: 10.1016/j.ijheatfluidflow. 2013.01.007.

[13] H. Wang, S. Xin, and P. Le Quéré, "Numerical study of natural convection-surface radiation coupling in air-filled square cavities," Comptes Rendus de Mecanique, vol. 334, no. 1, pp. 48-57, 2006. DOI: 10.1016/j. crme.2005.10.011.

[14] F. Archambeau, N. Méchitoua, and M. Sakiz, "Code saturne: A finite volume code for the computation of turbulent incompressible flows-industrial applications," Int. J. Finite Volumes, vol. 1, no. 1, 2004. https:// ijfv.math.cnrs.fr/spip.php?article3

[15] W. Fiveland, "Discrete-ordinates solutions of the radiative transport equation for rectangular enclosures," J. Heat Transfer, vol. 106, no. 4, pp. 699-706, 1984. DOI: 10.1115/1.3246741.

[16] M. F. Modest, Radiative Heat Transfer, 3rd ed. Boston, MA, USA: Academic Press, 2013.

[17] Y. Billaud, D. Saury, and D. Lemonnier, "Numerical investigation of coupled natural convection and radiation in a differentially heated cubic cavity filled with humid air. effects of the cavity size," Numer. Heat Transfer, A: Appl., vol. 72, no. 7, pp. 495-518, 2017. DOI: 10.1080/10407782.2017.1386509. 